\title{
Expositio missae :essai sur le commentaire du Canon de la Messe dans la tradition monastique et scolastique
}

Thèse de doctorat de l'Université de Bourgogne sous la direction de Vincent Tabbagh, octobre 2002

\section{Alain Rauwel}

\section{(2) OpenEdition}

\section{Journals}

Édition électronique

URL : https://journals.openedition.org/cem/3752

DOI : $10.4000 /$ cem. 3752

ISSN : 1954-3093

\section{Éditeur}

Centre d'études médiévales Saint-Germain d'Auxerre

Édition imprimée

Date de publication : 15 août 2003

ISSN : 1623-5770

\section{Référence électronique}

Alain Rauwel, «Expositio missae :essai sur le commentaire du Canon de la Messe dans la tradition monastique et scolastique », Bulletin du centre d'études médiévales d'Auxerre | BUCEMA [En ligne], 7 | 2003, mis en ligne le 05 décembre 2007, consulté le 22 septembre 2022. URL : http:// journals.openedition.org/cem/3752 ; DOI : https://doi.org/10.4000/cem.3752

Ce document a été généré automatiquement le 22 septembre 2022.

\section{(c) (i) 8 (O)}

Creative Commons - Attribution - Pas d'Utilisation Commerciale - Partage dans les Mêmes Conditions 4.0 International - CC BY-NC-SA 4.0

https://creativecommons.org/licenses/by-nc-sa/4.0/ 


\section{Expositio missae :essai sur le commentaire du Canon de la Messe dans la tradition monastique et scolastique}

Thèse de doctorat de l'Université de Bourgogne sous la direction de Vincent Tabbagh, octobre 2002

\section{Alain Rauwel}

1 Tout médiéviste qui s'intéresse à la vie religieuse des âges de Chrétienté sent que le discours historique de production courante ne fait pas la place assez belle à l'action liturgique. Il voit bien qu'à côté des occurrences extraordinaires, qui ont seules laissé des témoignages, on a tort de négliger ce qui fait en somme la trame de tout tissu religieux : ce que voit, ce qu'entend le fidèle qui vient à l'église, à chaque jour d'obligation, assister aux cérémonies du culte. C'est là, de toute évidence, un universel dont la réitération proprement infinie, au-delà des variantes de temps et de lieu - on dirait volontiers son éternel retour - n'a pu que marquer de façon déterminante le rapport au sacré dans le monde latin. Mais il s'agit, chez presque tous les historiens, d'une perception confuse. Comment, en effet, évaluer ce phénomène ? Comment mettre à l'épreuve l'idée de la centralité liturgique ? Le mieux ne serait-il pas d'abord de revenir (ou de venir...) aux textes dans lesquels les auteurs médiévaux ont élaboré leur conception du rite, du geste et de la parole efficaces?

2 Ces textes, largement négligés depuis le beau livre de Franz, vieux de tout juste un siècle ${ }^{1}$, j'ai choisi de leur donner le nom générique d'expositiones missae. Ce choix a été fait en parfaite conscience de la contradiction qu'il suppose avec les définitions de Dom Wilmart. Dans l'un de ces riches articles du Dictionnaire d'archéologie chrétienne et de liturgie qui valent un volume entier de recherches, le savant Bénédictin a en effet préconisé de réserver le vocable aux productions didactiques de l'âge carolingien ${ }^{2}$. Quelle que soit la pertinence des remarques proposées à l'appui de cette thèse, elle est intenable, dans la mesure où le $\mathrm{XI}^{\mathrm{e}}$ siècle ne représente en aucun cas une coupure 
absolue par rapport aux genres et aux thèmes antérieurs, ne serait-ce que parce que l'on continue à puiser sans réticence dans le prodigieux gisement amalarien. Il est exact, en revanche, que le terme d'expositio missae est restrictif pour les plus ambitieuses des "Sommes liturgiques" des XI ${ }^{\mathrm{e}}$-XIV siècles qui, en volume, consacrent beaucoup plus de développements au calendrier qu'aux rites des sacrements (Jean Belet ou Guillaume Durand ont composé des "Années liturgiques" avant la lettre). Toutefois, on ne se refusera pas la commodité de faire usage de ces deux mots simples et évocateurs.

3 Une autre question est bien légitime : pourquoi avoir pris comme unité d'étude, non la Messe dans son ensemble, mais le seul Canon? Si les liturgistes médiévaux avaient produit des explications linéaires de la Messe stricto sensu, de l'introït à l'“Ite missa est", la tâche aurait été tout à fait concevable. Mais les solennités de l'autel sont pour eux au moins autant prétexte que texte, et il est d'usage qu'ils rattachent aux différentes parties du Saint Sacrifice tous les points de théologie ou d'ecclésiologie possibles, jusqu'à un véritable vertige d'exhaustivité. Le Canon est ainsi généralement pris entre un commentaire du Credo qui s'étend souvent jusqu'à un résumé complet de la doctrine catholique, et un autre du Pater, susceptible de se développer sur plusieurs dizaines de folios ${ }^{3}$. De même, au début de la Messe, la description de la procession et de son déroulement donne lieu à un petit traité des ordres sacrés, considérés au fur et à mesure de leur entrée dans le sanctuaire. La matière devient donc quasi impossible à dominer entièrement dans son ampleur et son éparpillement, et il faut se résoudre à choisir. Le Canon est alors un lieu d'observation privilégié, puisqu'il est au sommet de la courbe sacrale et qu'il représente dans la cérémonie le moment d'intensité maximale, à la fois du point de vue du théologien et de celui du simple fidèle. Mots et gestes y sont d'une richesse suffisante pour que l'on s'y consacre exclusivement.

Reste la chronologie adoptée (mi XI ${ }^{e}-m i X^{2}{ }^{e}$ siècle). Elle pose moins de problèmes. Il faut souligner d'abord que les textes fondateurs du IX siècle ne sont pas ici étudiés pour eux-mêmes, mais seulement présentés à titre de sources auxquelles les générations suivantes ne cessent de revenir. Vouloir faire un point définitif sur les expositiones carolingiennes supposerait une recherche spéciale, fondée sur la collation de nombreux manuscrits selon les méthodes propres de la philologie carolingienne. Si en effet le dossier d'Amalaire est à peu près clair, grâce aux éditions du P. Hanssens ${ }^{4}$, les "petits" commentaires anonymes appellent encore bien des éclaircissements. Par exemple, les variantes et similitudes des nombreux documents commençant par "Missa a sancto Petro" ou "Missa pro multis causis" demanderaient à être relevées exhaustivement pour déterminer s'il s'agit d'une seule famille de textes ou de plusieurs traditions distinctes. De même, la chronologie relative des pièces n'est pas assez fermement établie. La sagesse veut donc que l'on fixe son point de départ au $\mathrm{XI}^{\mathrm{e}}$ siècle. Il y a là, dans la période qui va des premiers Grégoriens aux grands Scolastiques, une véritable cohérence - même s'il faut maintenir une bipartition forte, très sensible dans l'analyse des genres littéraires, entre temps du Cloître et temps de l'École ${ }^{5}$. À l'autre extrémité, le milieu du XIV ${ }^{\mathrm{e}}$ siècle ${ }^{6}$ donne le sentiment d'une vraie rupture. Au-delà des raisons avancées dans la thèse (le développement, dont Guillaume d'Occam est le symptôme beaucoup plus que l'instigateur, d'une conception nouvelle de la transparence du langage moins favorable à la pratique traditionnelle du commentaire), le lecteur des grandes œuvres $\mathrm{du} \mathrm{XV}^{\mathrm{e}}$ siècle ne peut manquer d'être sensible à une forte différence de ton. John Lydgate, et a fortiori Gabriel Biel ou Josse Clichtove, appartiennent à un autre monde que celui des maitres en sacra pagina: le studium a 
tendance à y être recouvert par l'oratoire ; la dévotion (au sens moderne du terme) se fait plus présente ; bref : l'élévation tend à prendre le pas sur l'explication, modifiant définitivement le paradigme.

5 Avant toute analyse, dans les cadres qui viennent d'être fixés, il s'agit de rassembler un corpus. Même si l'on dispose de quelques guides, la tâche n'est guère aisée. Les éditions modernes, à l'exception de quelques très grands noms comme Rupert de Deutz, Belet ou Durand, sont plus que rares. Et c'est ici qu'il faut refuser de se joindre au concert classique de lamentations sur les insuffisances de la Patrologie latine. D'abord parce que, si cette collection n'existait pas, une recherche comme celle-ci serait absolument impossible. Mais surtout parce que les éditions de Migne (c'est-à-dire la plupart du temps les grandes éditions d'Ancien Régime) ne sont pas si mauvaises qu'on le dit. Le cas d'Hildebert de Lavardin est à ce point emblématique : tout le monde s'accorde sur l'obscurité de nombreux passages des Versus de mysterio missae, mais on l'attribue généralement aux erreurs de Beaugendre, reprises par Migne. Or la comparaison entre le texte imprimé et un manuscrit de bonne note ne faisant pas partie de ceux qu'utilisa le premier éditeur ${ }^{7}$ révèle très peu de variantes, et insignifiantes. Il faut donc se résigner à ce que ce soit Hildebert lui-même qui soit confus ; comme l'avait bien vu Hauréau, "s'efforçant de dire beaucoup de choses en peu de mots, et gêné dans cet effort par les règles de la métrique, il est souvent obscur" ${ }^{8}$. La conclusion s'impose : il ne faut pas médire trop vite des éditions anciennes.

6 Quant aux textes demeurés manuscrits, on peut se demander s'il faut systématiquement souhaiter leur édition. Ce serait bien sûr utile pour les pièces les plus anciennes (le remaniement de Jean d'Avranches par Maurille de Rouen, Oxford, Bodl. lat. 843) ... ou les plus récentes, dans la mesure où la théologie du XIV siècle, postérieure à la clôture des grandes collections, est quasiment terra incognita. Pour prendre un exemple, Nicolas Trivet, assez bien connu pour ses dispositions préhumanistes et ses commentaires d'œuvres classiques, mériterait que l'on aille, de l'Angleterre à l'Espagne, confronter les différents manuscrits de son Liber de officio missae. Pour d'autres auteurs, la peine et le coût d'une publication intégrale ne sont peut-être pas inévitables. Ainsi, une telle proportion de la grande Summa de officiis ecclesiasticis de Guillaume d'Auxerre est passée chez Durand qu'une bonne édition du Spéculateur et une table de concordance soignée peuvent suffire ${ }^{9}$. Enfin, des auteurs mineurs jusqu'ici négligés ne l'ont peut-être pas été sans raisons. Hervé de Bourg-Dieu, dont l'Expositio missae était jusqu'alors inédite, s'est révélé un pur recopieur du Liber officialis d'Amalaire. De même, Pierre de Roissy en la version longue de son Manuale n'a guère fait autre chose que reprendre en les développant de manière peu justifiée les thèmes énoncés avec une "brevitas romana" des plus caractéristiques dans le De missarum mysteriis d'Innocent III ${ }^{10}$. De telles découvertes sont intéressantes par ce qu'elles révèlent de l'influence exercée par quelques maîtres dominants, mais elles incitent aussi le philologue à faire sien le jugement pessimiste énoncé, il y a deux siècles, par Bréquigny : "l'examen des manuscrits encore ensevelis dans la poussière ne prouve que trop souvent qu'ils ne sont demeurés dans les ténèbres que parce qu'ils méritent peu de voir le jour" ${ }^{11}$...

7 Au total, ce dont le chercheur peut disposer commodément, entre Migne, la Continuatio medievalis et les manuscrits de Paris et de Rome, recouvre tout de même une bonne part du corpus. Sur la grosse cinquantaine de textes analysés, peu exigent des reprises complètes dans un avenir proche. Le dossier dont la réouverture serait la plus 
fructueuse est sans doute celui d'Honorius Augustodunensis. Ce polygraphe de première importance, qui est une des meilleures chambres d'écho de la tradition comme un des réservoirs de citations les plus fréquentés, a besoin d'éditions critiques, comme l'a montré V. Flint ${ }^{12}$.

8 Quels sont, maintenant, les apports proprement historiques des textes rassemblés ? Il faut dire d'abord ce qu'ils ne sont pas. Les expositiones n'apportent pas grand'chose à la connaissance des rites liturgiques dans leur matérialité. Les cérémonies de la Messe sont bien définies grâce aux très nombreux travaux d'érudition à elles consacrés depuis plusieurs siècles, et on ne peut plus espérer découvrir à leur propos beaucoup de nouveaux éléments. Le Canon, à plus forte raison, étant la partie de la célébration la plus tôt fixée (la structure est en place dès le De sacramentis ambrosien), ne peut guère faire l'objet d'une "histoire médiévale" ${ }^{13}$. Tout au plus établira-t-on de menus faits (la disparition de la Nativité au "Unde et memores" dans le courant du XII ${ }^{e}$ siècle), ou montrera-t-on que la réflexion des liturgistes sur le donné présent dans les sacramentaires et les missels a pu avoir des effets en retour. Ainsi le recours systématique à la symbolique des nombres (trois de la Trinité ou cinq des Cinq Plaies) a-t-il certainement influencé l'alignement à trois ou à cinq de tous les signes de croix du Canon, même lorsque le texte n'y invitait pas. C'est notable, ce n'est pas massif.

En théologie sacramentaire, le rassemblement des faits dogmatiques inclus dans les commentaires produit des cohortes serrées. Mais ces faits ne remettent pas en cause les grandes lignes du schéma d'évolution tracé à partir des traités considérant ex professo la question eucharistique. On a un temps estimé que l'évêque Étienne d'Autun, dans son De sacramento altaris, avait été le premier à employer le terme de transsubstantiation. Mais la révision chronologique radicale imposée pour cette œuvre par le P. Van Den Eynde ${ }^{14}$ a ruiné cette hypothèse. On peut cependant constater que les liturgistes ne tardent jamais à adopter le vocabulaire ou l'arsenal conceptuel des théologiens, et que leurs textes sont pour les élaborations doctrinales de bons moyens de diffusion : que l'on pense par exemple à la très efficace explication de la trinité res tantum / sacramentum et res / sacramentum tantum proposée par Lothaire de Segni et reprise par tous ses continuateurs. Rien d'étonnant à cela, d'ailleurs, dans la mesure où liturgistes et théologiens ne constituent pas deux groupes distincts, mais se confondent le plus souvent : la science liturgique, par bien des aspects, est une théologie appliquée, comme il arrive à la théologie d'avoir des implications très pratiques, dont la célèbre question eucharistique "Quid sumit mus" est l'emblème.

10 Diffusion, ai-je écrit : auprès du peuple chrétien ? En d'autres termes, y a-t-il entre $\mathrm{XI}^{\mathrm{e}}$ et $\mathrm{XIV}^{\mathrm{e}}$ siècle quelque chose qui pourrait, de près ou de loin, être assimilé à une "pastorale liturgique" ? La réponse est largement négative. Un sondage dans la masse énorme des sermons donne des résultats ambigus. Les rites de la Messe ne sont pas un sujet tabou, il arrive aux prédicateurs d'y consacrer à l'occasion un développement, mais au total le fait est rare, très loin de l'enthousiasme que révèle parallèlement le développement de la dévotion au Corpus Christi ${ }^{15}$. Sans doute quelque chose de la discipline de l'arcane subsiste-t-il dans une telle attitude, même si Jean Belet (XII ${ }^{e}$ siècle) est pratiquement le seul auteur à refuser tout commentaire écrit sur le Canon au motif que ce savoir doit être réservé aux prêtres. Surtout, il faut faire place à une assez abondante littérature en langue vernaculaire, qui indique la contenance à adopter pendant les cérémonies et les pieuses pensées à cultiver lors de leurs différentes étapes ; conformément à son genre et à son public, elle fait son apparition très avant dans le 
$\mathrm{XIII}^{\mathrm{e}}$ siècle, reste modeste au $\mathrm{XIV}^{\mathrm{e}}$ siècle et ne suscite une quantité vraiment significative de textes qu'au $\mathrm{XV}^{\mathrm{e}}$ siècle, donc hors des limites du présent travail. Toutefois, on rencontre dans les manuscrits d'expositiones, dès les années 1200, des prières à l'élévation très proches de celles qui abondent plus tard dans les livres d'heures.

11 Les remarques précédentes permettent de poser enfin ce qui semble, au bout du compte, la question la plus intéressante, même si ce n'est pas à coup sûr celle que l'on pouvait prévoir en commençant : quelle vision du sacerdoce dans ces traités écrits par des "professionnels" du sanctuaire à destination prioritaire de leurs semblables ? Quel prêtre pour célébrer chaque jour un Canon si minutieusement décrit et expliqué ? Les expositiones constituent un excellent point d'observation pour suivre l'émergence et la valorisation de la figure sacerdotale comme figure distincte, éminente, séparée (segregata), et cela précisément à cause du lien très fort entre caractère sacerdotal et célébration eucharistique. Ce thème mérite d'autant plus de retenir l'attention qu'il a été peu étudié jusqu'à présent. La littérature s'est concentrée sur la "cura animarum" dans sa dimension de gouvernement (Histoire des curés, ample bibliographie sur le sacerdoce des moines, etc.) ; à côté de ces recherches parfaitement légitimes, il y a place sans doute pour une autre histoire du prêtre latin au Moyen Âge, qui conjugue périodisation des éléments constitutifs du traité De Sacramento ordinis et rassemblement des traits d'une véritable spiritualité sacerdotale en formation. La pureté en est une composante essentielle, comme le montre le retour régulier de la question du "sacerdos pollutus", sur lequel on n'insiste tant que parce qu'il est l'anti-grégorien par excellence. Le bon prêtre, en revanche, est semblable à Aaron et aux prêtres de l'Ancienne Loi, zélés dans l'accomplissement des rites sacrificiels, qui fournissent aux auteurs du XII siècle, Hildebert de Lavardin en tête, leur comparaison privilégiée. Dans la perspective de cette référence au sacerdoce lévitique, l'onction sacramentelle prend une importance toujours plus grande, faisant presque disparaître le corps du prêtre au profit exclusif de ses mains - ses mains ointes, étendues sur les oblats pour en assurer la transsubstantiation. L'officium sacerdotale est avant tout le geste de bénédiction, écrit le Franciscain Guibert de Tournai au premier livre de son De officio episcopi ${ }^{16}$. Pour éviter toute discussion sur la validité des consécrations opérées par le ministère d'indignes, on en est venu ainsi à objectiver complètement l'action du ministre, qui n'est plus que cette "persona generalis" dont parle Alexandre de Hales ${ }^{17}$, bonne seulement à se faire le vecteur de la puissance d'un Autre. Mais une telle déperdition d'identité personnelle a pour contrepartie favorable un considérable accroissement d'identité collective, et surtout l'ouverture aux comparaisons les plus flatteuses : celui qui engendre chaque jour le Sauveur à l'autel n'est-il pas digne d'être rapproché de celle qui L'engendra à la crèche ? N'y aurait-il pas même, dans son pouvoir indéfiniment réitérable, une forme de supériorité sur la Vierge-Mère ? Ces questions sont inévitablement suscitées par l'analyse des expositiones missae. Mais on ne saurait espérer trouver toutes les réponses dans des textes riches, certes, mais aux finalités très précisément déterminées. Il reste beaucoup de sermons, beaucoup de recueils de prières privées à inventorier avant d'énoncer sans anachronisme ce que fut, en régime de Chrétienté, l'idéal sacerdotal chrétien. 


\section{NOTES}

1.A. FRANZ, Die Messe im deutschen Mittelalter : Beiträge zur Geschichte der Liturgie und des religiösen Volkslebens, Fribourg-en-Br., 1902.

2.A. WILMART, "Expositio missae", DACL, t. 5, col. 1014-1027.

3.Voir le cas typique de Bernard de Parentis dans le ms BnF nouv. acq. lat. 1733. Il y a lieu de se demander s'il ne faudrait pas chercher dans les expositiones l'une des matrices du Catéchisme du Concile de Trente et, à sa suite, de tous les grands catéchismes catholiques modernes, construits, comme on sait, sur une quadripartition dont au moins deux éléments sont présents dans notre corpus : l'élucidation du Symbole verset par verset au début, et celle de l'Oraison dominicale à la fin. 4.Amalarii episcopi opera liturgica omnia, Rome, 1948-1950 (Studi e testi, 138-140). 5.C'est incontestablement une des leçons de cette étude que la confirmation de la valeur à accorder au couple théologie monastique / théologie scolastique, un peu négligé ces dernières années. Si Rupert de Deutz et Hugues de Saint-Cher disent (peutêtre) la même chose, il est surtout évident qu'ils ne la disent pas de la même façon, et qu'on ne saurait aligner sur l'ordre rigoureux des quaestiones la théologie cordiale, scripturaire, ouverte à l'effusion, des grands Bénédictins du XII ${ }^{e}$ siècle.

6.Entendu, cela va de soi, avec une certaine souplesse : l'auditeur de Rote Arnaud Terreni, qui écrit en Avignon vers 1370, répond à tous les critères requis pour être intégré dans le corpus.

7.La copie conservée à la bibliothèque de Cîteaux, aujourd'hui Dijon 151.

8.Notices et extraits, t. 38/2, p. 430.

9.Cf. R. Martineau, "La Summa de officiis ecclesiasticis de Guillaume d'Auxerre", Études d'histoire littéraire et doctrinale du XIII ${ }^{\mathrm{e}}$ siècle, t. 2, Ottawa, 1932.

10.Cf. p. 275 de ma thèse la concordance entre les f. 30 v. -39 v. du ms BnF nouv. acq. lat. 232 et les livres III et IV d'Innocent.

11.Cité par J.-M. MOEGLIN, “L'Historiographie moderne et contemporaine en France et en Allemagne et les chroniqueurs du Moyen Âge", Saint-Denis et la royauté : études offertes à B. Guenée, Paris 1999, p. 317.

12."No firm analysis of Honorius's works can be made until they are edited afresh", "The Place and purpose of the works of Honorius Augustodunensis", Revue bénédictine, t. 87, 1977, p. 97.

13. À la différence de l'Offertoire, par exemple, où les différences locales ont été nettement plus sensibles, au moins jusqu'au concile de Trente : cf. les sondages effectués dans les premiers missels imprimés par Dom J.-M. POMMARES, Trente et le Missel, Rome 1997 (Bibliotheca Ephemerides Liturgicae, Subsidia, 94), ch. 1.

14. "Le Tractatus de sacramento altaris faussement attribué à Étienne de Baugé", Recherches de théologie ancienne et médiévale, t. 19, 1952, p. 225-243.

15.Sur ce dernier thème, on n'a jamais dépassé les belles études de E. DUMOUTET, Le Christ selon la chair et la vie liturgique au Moyen Âge, Paris, 1932.

16. Maxima bibliotheca veterum patrum, t. 25, p. 402.

17.Cf. B. MARLIANGEAS, Clés pour une théologie du sacerdoce : in persona Christi, in persona Ecclesiae, Paris, 1978 (Théologie historique, 51), p. 74. 
INDEX

Mots-clés : expositio missae, Canon de la messe, messe 